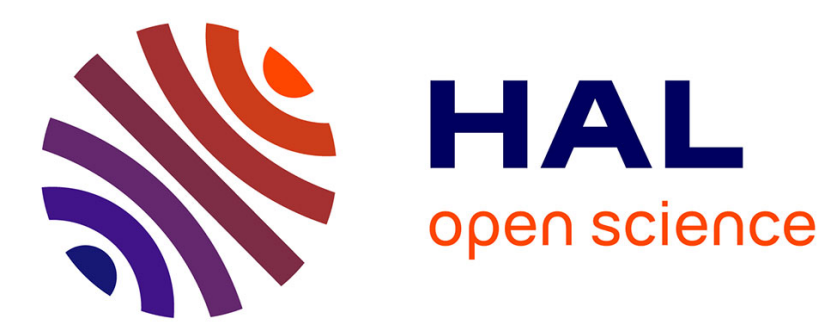

\title{
A high order moment method for the simulation of polydisperse two-phase flows
}

Aymeric Vié, Frédérique Laurent, Marc Massot

\section{To cite this version:}

Aymeric Vié, Frédérique Laurent, Marc Massot. A high order moment method for the simulation of polydisperse two-phase flows. Comptes Rendus Mécanique, 2013, Combustion, Flow and Spray Dynamics for Aerospace Propulsion, pp.1-10. 10.1016/j.crme.2012.10.004 . hal-00773327

\section{HAL Id: hal-00773327 \\ https://hal.science/hal-00773327}

Submitted on 13 Jan 2013

HAL is a multi-disciplinary open access archive for the deposit and dissemination of scientific research documents, whether they are published or not. The documents may come from teaching and research institutions in France or abroad, or from public or private research centers.
L'archive ouverte pluridisciplinaire $\mathbf{H A L}$, est destinée au dépôt et à la diffusion de documents scientifiques de niveau recherche, publiés ou non, émanant des établissements d'enseignement et de recherche français ou étrangers, des laboratoires publics ou privés. 


\title{
A high order moment method for the simulation of polydisperse two-phase flows
}

\author{
Aymeric Vié ${ }^{\mathrm{a}, \mathrm{b}}$, Frédérique Laurent ${ }^{\mathrm{a}, \mathrm{b}}$ Marc Massot ${ }^{\mathrm{a}, \mathrm{b}, \mathrm{c}}$ \\ ${ }^{a}$ CNRS, UPR 288 - "Laboratoire d'Énergétique Moléculaire et Macroscopique, Combustion" (EM2C), Grande Voie des \\ Vignes, 92295 Châtenay-Malabry, FRANCE \\ ${ }^{\mathrm{b}}$ Ecole Centrale Paris (ECP), Grande Voie des Vignes, 92295 Châtenay-Malabry, FRANCE \\ ${ }^{\mathrm{c}}$ Center for Turbulence Research, Stanford University, California 94305-4035, USA \\ Received $* * * * * ;$ accepted after revision +++++
}

\begin{abstract}
In this work, we are interested in the modeling of spray polydispersion in size as well as size-velocity correlations, which may greatly influence the evaporation and the dynamics of the disperse phase. Vié et al. 2011 proposed a new model called Coupled Size-Velocity Moment method (CSVM), which handles the polydispersion using the NDF reconstruction proposed in Kah at al. 2011 for size distribution, a velocity reconstruction and adapted numerical methods for moments evolution. Here, the CSVM is evaluated in a Frozen Homogeneous Isotropic Turbulence. Results shows the ability of the method to capture the statistical information of a turbulent flow with a minimal number of moments. As soon as a numerical scheme for unstructured grid is provided, the CSVM would be an interesting approach for complex simulations in industrial codes.
\end{abstract}

\section{Résumé}

Une méthode de moments d'ordre élevé pour la simulation d'écoulements diphasiques polydisperses. Dans ce travail, nous nous intéressons à la modélisation de la polydispersion en taille des sprays et la prise en compte des corrélations taille-vitesse, qui peuvent grandement influencer l'évaporation et la dynamique de la phase dispersée. Vié et al. 2011 ont proposé un nouveau modèle appelé Coupled Size-Velocity Moment method (CSVM), qui prend en compte la polydispersion avec la stratégie de reconstruction de la NDF proposée dans Kah et al. 2011 pour la distribution en taille, une reconstruction de la vitesse, et des méthodes numériques adaptées pour l'évolution des moments. Ici, le CSVM est évalué sur une Turbulence Homogène Isotrope Figée. Les résultats montrent la capacité de la méthode à capturer toutes les caractéristiques importantes de ce type d'écoulement avec un nombre minimal de moments. Dès qu'un schéma pour maillages non-structurés sera proposé, le CSVM deviendra une approche intéressante pour des simulations complexes avec des codes industriels.

Key words: Polydisperse sprays; high order moment method ; Entropy Maximization ; size-velocity correlations ; Homogeneous Isotropic Turbulence

Mots-clés : Sprays polydisperses ; méthode de moments d'ordre élevé; Maximisation d'Entropie; Corrélations taille-vitesse; Turbulence Homogène Isotrope 


\section{Introduction}

As numerous industrial applications involve the injection of a liquid fuel in a gaseous flow field (automotive engines, aeronautical combustors, industrial furnaces...), dedicated modeling and numerical methods are expected in order to provide predictive simulations. However the full injection process experiences several physics which are difficult to simulate simultaneously, and which result in a disperse liquid phase.

In this work, we are interested in the simulation of this disperse liquid phase resulting from the atomization of the liquid jet. In this perspective, Eulerian methods seem really interesting for several reasons: ease of parallelization, coupling with the Eulerian gas phase and a more natural coupling with the primary atomization methods than Lagrangian methods [1]. For monodisperse (one size per position and time) and monokinetic (one velocity per position and time), two-fluids models or Equilibrium Eulerian approaches can be used (for a review, see [1]). But as the physics of the disperse phase become more and more complex, the modeling effort increases. The literature tends to address separately two main issues: the polydispersion in size and the multi-velocity aspects. Concerning the multi-velocity for monodisperse flows, some works are dedicated to the effects of the turbulence on a disperse liquid phase $[2,3,4,5]$ by means of an uncorrelated energy which acts as additional pressure and diffusion in the limit of close-toequilibrium velocity distributions. For far-from-equilibrium and trajectory crossings aspects, the problem is addressed by moments methods with quadrature closures $[6,7,8]$, and in $[9,10]$ the authors also propose a new approach that aims at describing both issues using a Multi-Gaussian quadrature. Concerning the polydispersion in size, three types of method can be envisaged: Multifluid approaches which divide the size space into sections [11, 12, 13], moment methods with quadrature closures [14, 15], and moment methods with continuous NDF reconstruction [16, 17]. Some others studies had also tried to address polydispersion and multi-velocity aspects in a common framework $[18,19,20]$.

To describe size polydispersion, the Eulerian Multi-Size Moment method (EMSM) of Kah et al. [16, 17],which is an hybrid approach taking advantage of high order moments as well as a discretization in size space to describe the NDF, has demonstrated its efficiency compared to others methods. But this method still has one drawback: it considers one velocity for all sizes, which would be too restrictive for wide Stokes number spectrum. Recently, Vié et al. [21] have developed the Coupled Size-Velocity Moment method (CSVM), which introduces the description of the velocity conditioned by the size in the NDF reconstruction. The authors demonstrates the ability of such approaches to reproduce size-conditioned dynamics even in extreme pathological configurations like the injection in a crossflow or the Taylor-Green Vortices, in order to assess the robustness of such an approach, and in more realistic configurations such as the weakly turbulent free jet. Furthermore, the CSVM method keeps the hybrid aspect of the EMSM, as multiple size intervals can be used to describe the NDF and the velocity conditioned by size.

In this work, the aim is to reach a higher level of validation, by investigating precise statistical informations. For this purpose, the proposed test case is a Frozen Homogeneous Isotropic Turbulence (HIT), in which we can examine important quantities such as segregation or mean size variance, which are of primary importance for evaporation, combustion or ignition. Results are compared to the 10 sections Multifluid, which captures the size-velocity correlations in the sense that every section has its proper velocity, but does not handle size-conditioned velocities inside each section. It is shown that the CSVM is able to reach the same level of accuracy as the Multifluid Approach using only 8 moments, compared to the 30 moments of the Multifluid (in $3 \mathrm{D}$, it will be 10 versus 40 ).

The paper is organized as follows. First, we present the CSVM method, arguing on the differences with the EMSM, and the difficulties that arise from the new description of the kinetic level. Then we will apply this method to a Frozen HIT, and compare the results to a Multifluid approach which naturally accounts

Email addresses: aymeric.vie@em2c.ecp.fr (Aymeric Vié), frederique.laurent@em2c.ecp.fr (Frédérique Laurent), marc.massot@em2c.ecp.fr (Marc Massot). 
for a partial information of the size-velocity correlations and the EMSM which has only one velocity for all sizes.

\section{The Coupled Size-Velocity Moment method (CSVM)}

In this section, the CSVM method is introduced. The moment problem that we want to solve is detailed with the two main issues that arise. The reconstruction strategies for the velocity and number density distributions against size, and the dedicated numerical methods that are used for the evolution of the moments set are finally presented.

\subsection{The moment problem}

We consider dilute sprays, so that there is no effect of the dispersed phase on the gas phase, and high Knudsen numbers, so that collisions can be considered with a very good approximation as negligible. The phase space of the NDF is limited to size $S$ and velocity $v$ in one dimension, leading to the following non-dimensional Population Balance Equation (PBE):

$$
\frac{\partial f}{\partial t}+v \frac{\partial f}{\partial x}+\frac{\partial}{\partial v}\left(\frac{u_{g}-v}{\mathrm{St}} f\right)+\frac{\partial}{\partial S}\left(R_{S} f\right)=0
$$

where $R_{S}=\mathrm{d} S / \mathrm{d} t$ is the evaporation rate which is constant in the case of $d^{2}$ evaporation laws and $\mathrm{St}=\tau_{p} / \tau_{g}$ is the Stokes number, i.e. the ratio between the relaxation time of the droplets and a reference timescale $\tau_{g}$ for the gas phase. To solve this equation, we are looking at the moments of the NDF. In one dimension, these are defined by:

$$
M_{i}^{l}(t, x)=\int_{-\infty}^{\infty} \int_{0}^{1} S^{l} v^{i} f(t, x, v, S) \mathrm{d} v \mathrm{~d} S .
$$

The equation for $M_{i}^{l}(t, x)$ is then:

$$
\frac{\partial M_{i}^{l}}{\partial t}+\frac{\partial M_{i+1}^{l}}{\partial x}=-\int_{0}^{1} i \frac{S^{l}}{\mathrm{St}}\left(v^{i}-u_{g} v^{i-1}\right) f \mathrm{~d} S \mathrm{~d} v+R_{S} M_{i}^{l-1}+R_{S}\left[\int_{-\infty}^{\infty} v^{i} S^{l} f(t, x, v, S) \mathrm{d} v\right]_{S=0}^{S=1} .
$$

In this equation, the main issues concern the modeling of the second left hand side term (convection) and the third right hand side term (evaporation). The former is due to the fact that with a finite set of moments, the flux for the highest order moment equation is unclosed. The latter imposes the knowledge of point-wise values of the NDF at the edge of the size phase space to determine fluxes in size space, the sole contribution being the flux at zero size (there is no flux at $S=1$, as the evaporation decreases the sizes, and there is no droplets beyond $S=1$ ), which corresponds to a disappearance rate of droplets that reach a zero size [17].

In order to close Eq. 3, a presumed shape for the NDF is needed. In the CSVM, as we aim at the description of the size-conditioned dynamics, we will consider a unique velocity for each size:

$$
f(t, x, v, S)=n(t, x, S) \delta(v-U(t, x, S)),
$$

where $n$ and $U$ depend on position and time, but also on droplet surface. At this point, a reconstruction method is needed to recover $n(t, x, S)$ and $U(t, x, S)$ from the knowledge of the moments.

\subsection{Strategy for size reconstruction}

The determination of $n(t, x, S)$ is achieved using size moments $M_{0}^{l}=\int_{0}^{1} S^{l} n(t, x, S) d S$, so $U(t, x, S)$ is not needed at this point. In this work we will considered four moments in size, but the method can 
be extended to more moments. The CSVM uses the reconstruction strategy of the EMSM: $n(t, x, S)$ is reconstructed using Entropy Maximization (EM)[22, 17]. The Shannon Entropy is defined by:

$$
\mathbf{H}(f)=-\int_{0}^{1} n(t, x, S) \ln n(t, x, S) \mathrm{d} S .
$$

Associated with $N$ moments constraints, the maximization of $\mathbf{H}(f)$ imposes the unique following reconstruction:

$$
n(S)=\exp \left(-\sum_{j=0}^{N} \zeta_{j} S^{j}\right)
$$

where $\zeta_{j}$ are Lagrange multipliers. The following convex potential is then minimized:

$$
\Delta=\int_{0}^{1}\left[\exp \left(-\sum_{j=0}^{N} \zeta_{j} S^{j}\right)-1\right] \mathrm{d} S+\sum_{j=0}^{N} \zeta_{j} M_{0}^{j} .
$$

Indeed, its stationary points are given by:

$$
\frac{\partial \Delta}{\partial \zeta_{i}}=0 \Rightarrow \int_{S_{\min }}^{S_{\max }} S^{i} \exp \left(-\sum_{j=0}^{N} \zeta_{j} S^{j}\right) \mathrm{d} S=M_{0}^{i} .
$$

Numerically, this non-linear system is solved using a Newton method: starting from initial choices $\zeta=$ $\left(\zeta_{0}, \ldots, \zeta_{N}\right)^{T}$, updated $\zeta$ is defined from:

$$
\zeta^{+}=\zeta-H^{-1}\left(\mathcal{M}_{0}-\langle X\rangle_{\zeta}\right)
$$

where $\langle X\rangle_{\zeta}=\left(\left\langle x^{0}\right\rangle_{\zeta}, \ldots,\left\langle x^{N}\right\rangle_{\zeta}\right)^{T}$ is the vector of approximated moments:

$$
\left\langle x^{i}\right\rangle=\int_{0}^{1} x^{i} \exp \left(-\sum_{j=0}^{N} \zeta_{j} x^{j}\right) d x
$$

and $H$ is the Hessian matrix defined by $H_{i, j}=\frac{\partial \Delta}{\partial \zeta_{i} \partial \zeta_{j}}=\left\langle x^{i+j}\right\rangle_{\zeta}$ for $i, j=0, . ., N$. In [21], three ingredients are added to the EM, compared to the initial work of Massot et al [17]. First, to be able to compute the integrals of any NDF, a Gauss-Legendre quadrature with an adaptive support is proposed in [21] and allows to reach a high accuracy with a minimal number of quadrature points (here 20 points are used). Second, a tabulated initial guess is used to increase the convergence rate of the Newton solver. Finally, the number of parameters of the EM is adapted at the border of the moment space ${ }^{1}$ within a controlled error.

\subsection{Strategy for velocity reconstruction}

For velocity reconstruction, size-velocity moments at first order in velocity are used. These moments depend on the reconstruction of $n(t, x, S)$, so the size reconstruction must be performed before the velocity reconstruction. Hereafter, the reconstruction strategy for one dimension is proposed. In $2 \mathrm{D}$ or $3 \mathrm{D}$, the

1. The moment space is a subspace of $\mathbb{R}^{N+1}$ where a moment vector can lead to an infinity of positive and realizable NDF, which is called the Haussdorf finite moment problem. At the border of the moment space, the NDF is uniquely determined, and corresponds to a measure solution, i.e. a sum of Dirac's $\delta$-functions. 
reconstruction in one direction does not depend on the velocity of the other directions, so the multidimensional strategy is straightforward. The size-velocity moments are:

$$
M_{1}^{l}=\int_{0}^{1} S^{l} U(S) n(S) \mathrm{d} S, \quad l=0, N_{v}-1 .
$$

where $N_{v}$ is the number of size-velocity moments that are used. To evaluate the velocity of each size, a reconstruction shape of $U(t, x, S)$ is proposed. A constraint on this reconstruction is that droplets with a null size follow the gas phase: $U(t, x, S=0)=u_{g}$. Furthermore, to be able to represent all possible moment vectors, it is necessary to propose a reconstruction that can describe the full velocity moment space. The following power reconstruction is proposed ${ }^{2}$ :

$$
U(t, x, S)=u_{g}+\sum_{k=1}^{N_{v}} A_{k} S^{\alpha_{k}},
$$

where $A_{k}$ are the parameters used to control the size-velocity moments, and $\alpha_{k}$ are user-determined with $\alpha_{1}<\alpha_{2}<\ldots<\alpha_{N}$. Then, one can write:

$$
M_{1}^{l}=M_{0}^{l} u_{g}+\sum_{k=1}^{N_{v}} A_{k} \int_{0}^{1} S^{\alpha_{k}+l} n_{l}(S) \mathrm{d} S,
$$

which gives the linear system for the parameters vector $\mathcal{A}=\left(A_{1}, \ldots, A_{N_{v}}\right)$ :

$$
P \mathcal{A}=\mathcal{N}, \quad P_{k l}=\int_{0}^{1} S^{\alpha_{k}+l} n_{l}(S) \mathrm{d} S, \quad N_{l}=M_{1}^{l}-M_{0}^{l} u_{g} .
$$

Considering the linear system, the only configuration for which this reconstruction will not be possible for a given moment vector corresponds to the zero determinant for matrix $P$. This condition is reached for a Dirac's $\delta$-function for the size distribution $n(S)$. As the EM does not generate this type of distribution ${ }^{3}$, our reconstruction strategy can reproduce any moment vector given by the proposed method. The sole issue is that $P$ may be badly conditioned where we are close to Dirac's $\delta$-function. But in fact, the determinant of $P$ becomes close to zero, and the problem is overdetermined, so that we can shift to a one-parameter reconstruction by checking the determinant value.

In this work, as the aim is to design a method with as few moments as possible, only one additional sizevelocity moment per dimension will be used, compared to the original EMSM. So we use a reconstruction with two parameters $\alpha_{1}=0.5$ and $\alpha_{2}=1$, which is proven sufficiently accurate to describe moment evolution, and is an optimal choice in terms of reconstruction error for a two moment reconstruction [21].

Finally, we emphasize on the fact that the proposed velocity reconstruction keep the hybrid aspect of the initial EMSM, meaning that we can use a discretization of the size space in a Multifluid context, as well as our high order moment description in each section. This is due to the fact that the linear system of the velocity reconstruction does not depend on the integration interval, but directly on moments. Furthermore, every size interval will keep an information of the gas phase velocity, even if the size interval does not contain the zero size, at which droplets are in equilibrium with the gas phase.

2. In [18], an exponential reconstruction is proposed, based on the analytical solution of the relaxation of droplets at constant gas velocity. Although this is a physical and relatively smooth reconstruction, with good asymptotic predictions, this shape is not able to reproduce all the moment space, as it cannot capture inversions in the relative velocity between gas and droplets, which is a typical case in turbulence.

3. The EM cannot reproduce Dirac's $\delta$-functions exactly, because of the presumed NDF, but can be as close as necessary from this type of distribution, under a given tolerance on moments. 


\subsection{Evaporation and drag force}

The strategy for the evolution of the NDF through evaporation and drag force is presented. The method described in [17] is based on two main steps: the evaluation of the disappearance flux of droplets, and the evolution in size space. The strategy is extended to the evolution in velocity space through drag force. The derivation is shown for $d^{2}$ law and Stokes drag only $\left(R_{S}=\right.$ cst and $\left.\operatorname{St}(S)=\operatorname{St}^{0} S\right)$, but the extension to complex drag and evaporation laws is straightforward, thanks to the quadrature step. The size reconstruction of the CSVM enables the computation of the disappearance flux of droplet $F_{i}^{l}$ for each moment and for one time step $\Delta t$ :

$$
F_{i}^{l}=\int_{0}^{-R_{S} \Delta t} U(S)^{i} S^{l} n_{l}(S) \mathrm{d} S,
$$

corresponding to the part of the distribution which reaches the zero size during one time step. The moments are then corrected:

$$
\tilde{M}_{i}^{l}=M_{i}^{l}-F_{i}^{l} .
$$

The evolution in phase space is determined by a CQMOM-like quadrature [7]:

$$
\tilde{M}_{i}^{l}=\sum_{k=1}^{2} w_{k} S_{k}^{l} U_{k}^{i}
$$

The evolution of the moments is done by evolving the weights and abscissas of the quadrature:

$$
\frac{\mathrm{d} U_{k}}{\mathrm{~d} t}=-\frac{1}{\mathrm{St}^{0} S_{k}}\left(U_{k}-u_{g}\right), \quad \frac{\mathrm{d} S_{k}}{\mathrm{~d} t}=R_{S} .
$$

The size and velocity after one time step are:

$$
\begin{aligned}
& S_{k}(t+\Delta t)=S_{k}(t)+R_{S} \Delta t \\
& U_{k}(t+\Delta t)=\left(U_{k}(t)-u_{g}(t)\right)\left(\frac{S_{k}(t)+R_{S} \Delta t}{S_{k}(t)}\right) \overline{\mathrm{St}^{0} R_{S}}+u_{g}(t) .
\end{aligned}
$$

with the approximation of a constant gas velocity during the time step. With the updated quadrature, the new moments can be computed:

$$
M_{i}^{l}(t+\Delta t)=\sum_{k=1}^{2} w_{k}(t) S_{k}(t+\Delta t)^{l} U_{k}(t+\Delta t)^{i} .
$$

In the case of $d^{2}$ law and Stokes drag, the quadrature approximation for the moments evolution is proven to be efficient $[17,21]$. For complex laws, this approach is still a good approximation of the real dynamics and a computationally efficient method, and keeps the moments inside the moment space.

\subsection{Convection scheme}

The physical space evolution is surely the most important part of the size-velocity correlations. As shown in [21], the constant velocity assumption, even if it leads to higher errors, does not lead to a completely different moment evolution in the velocity phase space. But in physical space, the dynamics will be significantly different. The moment equation for the convection in one dimension is:

$$
\frac{\partial \mathcal{M}}{\partial t}+\frac{\partial \mathcal{F}(\mathcal{M})}{\partial x}=0
$$


where $\mathcal{M}=\left(M_{0}^{0}, M_{0}^{1}, M_{0}^{2}, M_{0}^{3}, M_{1}^{0}, M_{1}^{1}\right)^{T}$ is the moment vector and $\mathcal{F}(\mathcal{M})=\left(M_{1}^{0}, M_{1}^{1}, M_{1}^{2}, M_{1}^{3}, M_{2}^{0}, M_{2}^{1}\right)^{T}$ is the flux vector. For a constant velocity distribution, the fluxes are simple functions of the moments. In this case, a first order finite-volume scheme preserves the moment space, as it is positive definite and the scheme reconstruction generates realizable moments. The second-order scheme that preserves the moment space is much more difficult to design, and this issue raised in the literature [23] had been finally tackled in [16]. In the case of a continuous non-constant velocity, fluxes are now a complex function of the moments, and a first solution has been proposed in [21], but has not yet been implemented in 2D.

The proposed scheme is based on a kinetic flux splitting at the semi-kinetic level, in order to obtain a positivity-preserving and monotone scheme. The convection of each droplet size is driven by the following infinite semi-kinetic system for $S=[0,1]$ :

$$
\begin{aligned}
\frac{\partial n(S)}{\partial t}+\frac{\partial n(S) U(S)}{\partial x} & =0, \\
\frac{\partial n(S) U(S)}{\partial t}+\frac{\partial n(S) U(S)^{2}}{\partial x} & =0 .
\end{aligned}
$$

To solve this system, a first order upwind finite volume scheme is used:

$$
\begin{aligned}
n_{j}^{n+1} & =n_{j}^{n}-\frac{\Delta t}{\Delta x}\left(F_{j+1 / 2}-F_{j-1 / 2}\right), \\
n_{j}^{n+1} U_{j}^{n+1} & =n_{j}^{n} U_{j}^{n}-\frac{\Delta t}{\Delta x}\left(G_{j+1 / 2}-G_{j-1 / 2}\right),
\end{aligned}
$$

where:

$$
\begin{aligned}
F_{j+1 / 2} & =\min \left(0, U_{j+1}^{n}\right) n_{j+1}^{n}+\max \left(0, U_{j}^{n}\right) n_{j}^{n}, \\
G_{j+1 / 2} & =\min \left(0, U_{j+1}^{n}\right) n_{j+1}^{n} U_{j+1}^{n}+\max \left(0, U_{j}^{n}\right) n_{j}^{n} U_{j}^{n} .
\end{aligned}
$$

By integrating over size, the following scheme is obtained:

$$
\mathcal{M}_{j}^{n+1}=\mathcal{M}_{j}^{n}-\frac{\Delta t}{\Delta x}\left(\mathcal{F}_{j+1 / 2}-\mathcal{F}_{j-1 / 2}\right),
$$

where the fluxes are splitted into positive and negative components:

$$
\mathcal{F}_{j+1 / 2}=\int_{0}^{1} \mathcal{S}\left(\min \left(0, U_{j+1}^{n}\right) n_{j+1} \mathcal{U}_{j+1}^{n}+\max \left(0, U_{j}^{n}\right) n_{j} \mathcal{U}_{j}^{n}\right) \mathrm{d} S=\mathcal{F}_{j+1}^{-}+\mathcal{F}_{j}^{+},
$$

where $\mathcal{S}=\left[1, S, S^{2}, S^{3}, 1, S\right]^{T}$ and $\mathcal{U}_{j}^{n}=\left[1,1,1,1, U_{j}^{n}, U_{j}^{n}\right]^{T}$. Using the size and velocity reconstructions these fluxes can be computed for every moments. To ensure the stability of the scheme, a CFL-like condition is imposed on the timestep, based on the maximum reconstructed velocity.

\section{Application to an Homogeneous Isotropic Turbulence}

The proposed test case is a $2 \mathrm{D}$ frozen homogeneous isotropic turbulence generated with the ASPHODELE code of CORIA, which solves 3D Low-Mach number gas phase Navier-Stokes equations [12]. The liquid phase is injected homogeneously in the domain at $t=0$ with the same velocity as the gas phase. The turbulence is generated following the Pope spectrum for which the parameters are set to $p_{0}=4$, $c_{L}=0.013, c_{\eta}=0.105$ and $\beta=5.2$ [24]. The droplet number density is constant and the Stokes numbers range based in the Kolmogorov time scale is $\mathrm{St} \in[0,1]$. Predicting this type of flows is important, as it is expected to exhibit the main effects of a turbulence gas field on a disperse liquid phase, i.e. the preferential concentration of particles in low vorticity zones which greatly influences for instance the 
combustion or auto-ignition and will be of primary importance for final applications. This effect is highly size-conditioned, so that this test case is particularly interesting for qualifying the accuracy of the method.

In Fig. 1, number density at time $t=3$ obtained with Lagrangian approach, EMSM, Multifluid with 10 sections and CSVM are compared. For the four approaches, the structures are very similar, even for EMSM which does not account for the size-velocity correlations. However it appears that the Lagrangian results are less diffuse than the Eulerian results. But at this point, we know that the Eulerian approaches that we are using raise two issues: the missing modeling for high Stokes flows, and the first order numerical scheme. The missing modeling is the velocity dispersion that is accounted for in [2], which is due to the existence of an uncorrelated component for the motion of droplet in high Stokes flow. Here we do not account for it, but this will be investigated in future work, based on the methods in [3, 10]. The diffusive effect of a low-order numerical scheme was also assessed in [5]. The second order scheme proposed in [21] will be tested in perspective of this work. But in fact, our results can be compared to the Multifluid approach with a first order scheme and no velocity dispersion, as this method captures naturally the effects that are modeled in the CSVM, even if the size-velocity correlations are not continuously reproduced.

To analyse further the liquid field, Fig. 2 compares the number density for small droplets (St $\in[0,0.1])$, medium droplets $(\mathrm{St} \in[0.5,0.6])$ and big droplets $(\mathrm{St} \in[0.9,1.0])$. Here it is clear that each section has its proper dynamics, as the segregation appears different and grows with the Stokes number. The CSVM and Multifluid approaches produce equivalent results whereas the EMSM, which has the same number density repartition for each size, cannot capture such size-conditioned dynamics.

To analyse the spatial dispersion of the particle in a qualitative way, we now examine the segregation of particles $G_{p p}=\left\langle\left(M_{00}^{0}\right)^{2}\right\rangle /\left\langle\left(M_{00}^{0}\right)\right\rangle^{2}$ and the spatial variance of the mean size $\sigma\left(S_{m}\right)=\sqrt{\left\langle S_{m}^{2}\right\rangle-\left\langle S_{m}\right\rangle^{2}}$ where $<$. $>$ denotes the spatial average over the full domain and $S_{m}=M_{00}^{1} / M_{00}^{0}$ is the mean size. The results are plotted in Fig. 3. First, we can notice that the EMSM, which sees only one droplet size with respect to the drag force, predicts a higher total segregation than CSVM and Multifluid, which see a polydisperse flow and produce the same results. More interesting, the sectional segregation (i.e. the segregation of each size intervals) are really similar for CSVM and Multifluid, for all sections. For the mean size variance, a perfect agreement between CSVM and Multifluid is also shown, demonstrating the ability of the method to capture a crucial information for evaporation and combustion.

To assess the accordance of CSVM and Multifluid approaches on local quantities, we investigate NDF and velocity distributions at three locations in the domain: in the center of a vortex where the number density is low, in a medium number density zone and in a high number density zone. The results are plotted in Fig. 4 and conclude on the full agreement between CSVM and Multifluid. In particular, the NDF is accurately captured by CSVM with only four moments in size, comparing to the ten size moments for Multifluid, and the velocity distributions are captured with only two moments per direction, comparing to the ten moments per direction of the Multifluid.

Finally, we will end by concluding on the computational time of our approach. For this case the CSVM needs 1580 seconds, whereas the 10 sections Multifluid needs 1260 seconds, so the method seem equivalent in terms of computational time. It remains that our method uses lower memory and storage space than Multifluid, which will be really important for implementation in industrial applications. To reduce this overcost comparing to the initial EMSM approach, two solutions are envisaged, which concern the computation of the integrals. Basically the computation of the moments and Jacobians matrices of the EM Newton Solver, and the fluxes of the convection scheme involves a Gauss-Legendre quadrature. Here, more than $80 \%$ of the computational time is spent in the computation of the integrals. To reduce this cost, a solution would be to reduce the number of moments to three and to use two sections instead of one. In this case, we have analytical solutions for the integrals, and the computational time would be significantly decreased. 

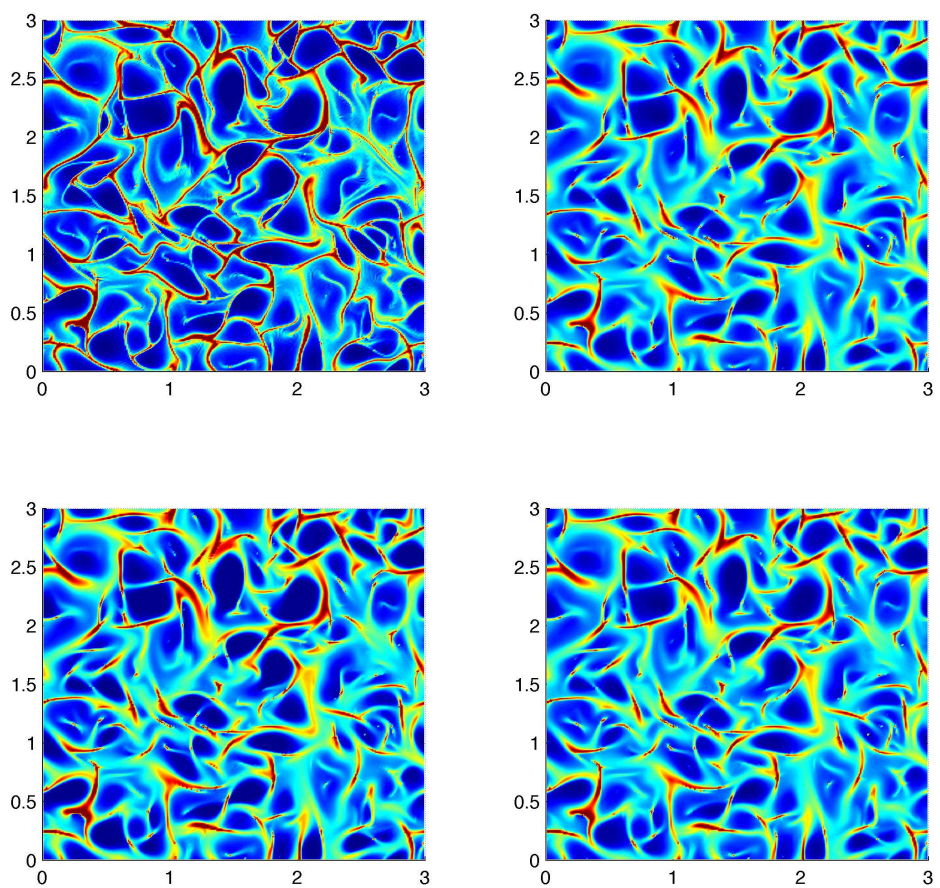

Figure 1. Droplet number density at time $t=3$ : Lagrangian (upper left), Multifluid 10 sections (upper right), EMSM (lower left), and CSVM (lower right).
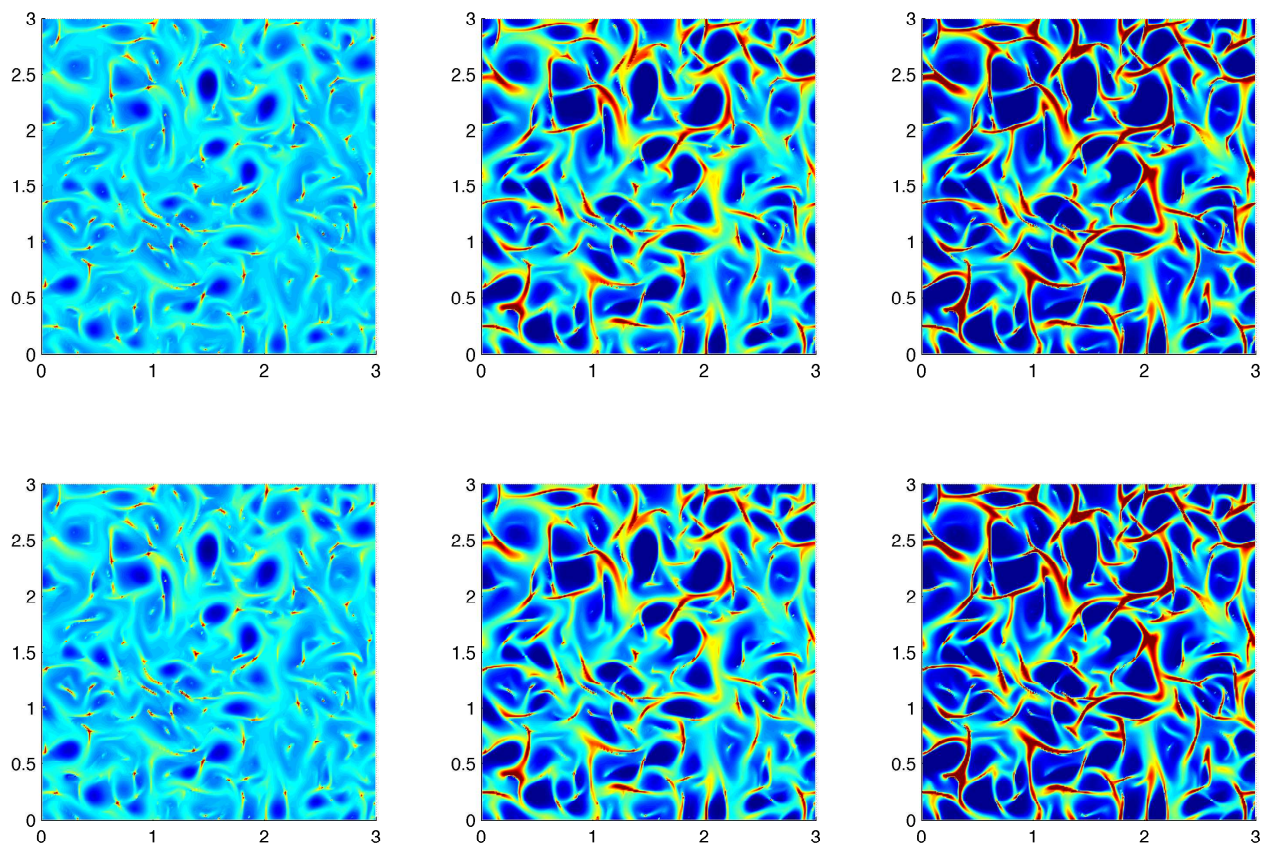

Figure 2. Droplet number density at time $t=3$ : Multifluid 10 sections (up) ad CSVM (down) for Stokes numbers St $\in[0,0.1]$ (left), St $\in[0.5,0.6]$ (center) and $\mathrm{St} \in[0.9,1.0]$ (right). 

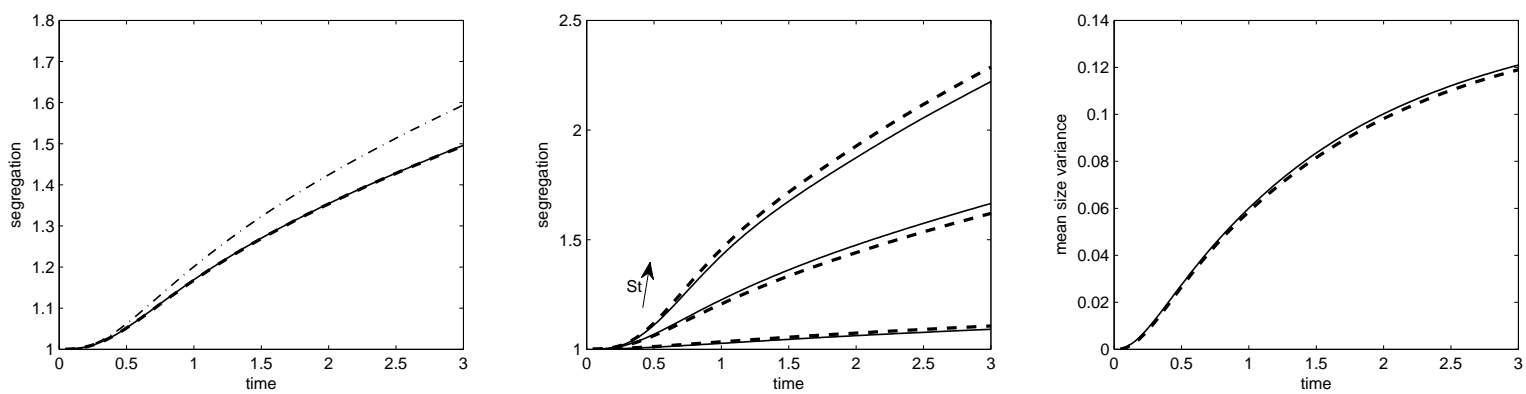

Figure 3. Total segregation (left), sectional segregation (center) and spatial mean size variance (right) against time: EMSM (dot-dashed line), Multifluid 10 sections (full line) and CSVM (dashed line). The arrow indicates growing Stokes numbers.
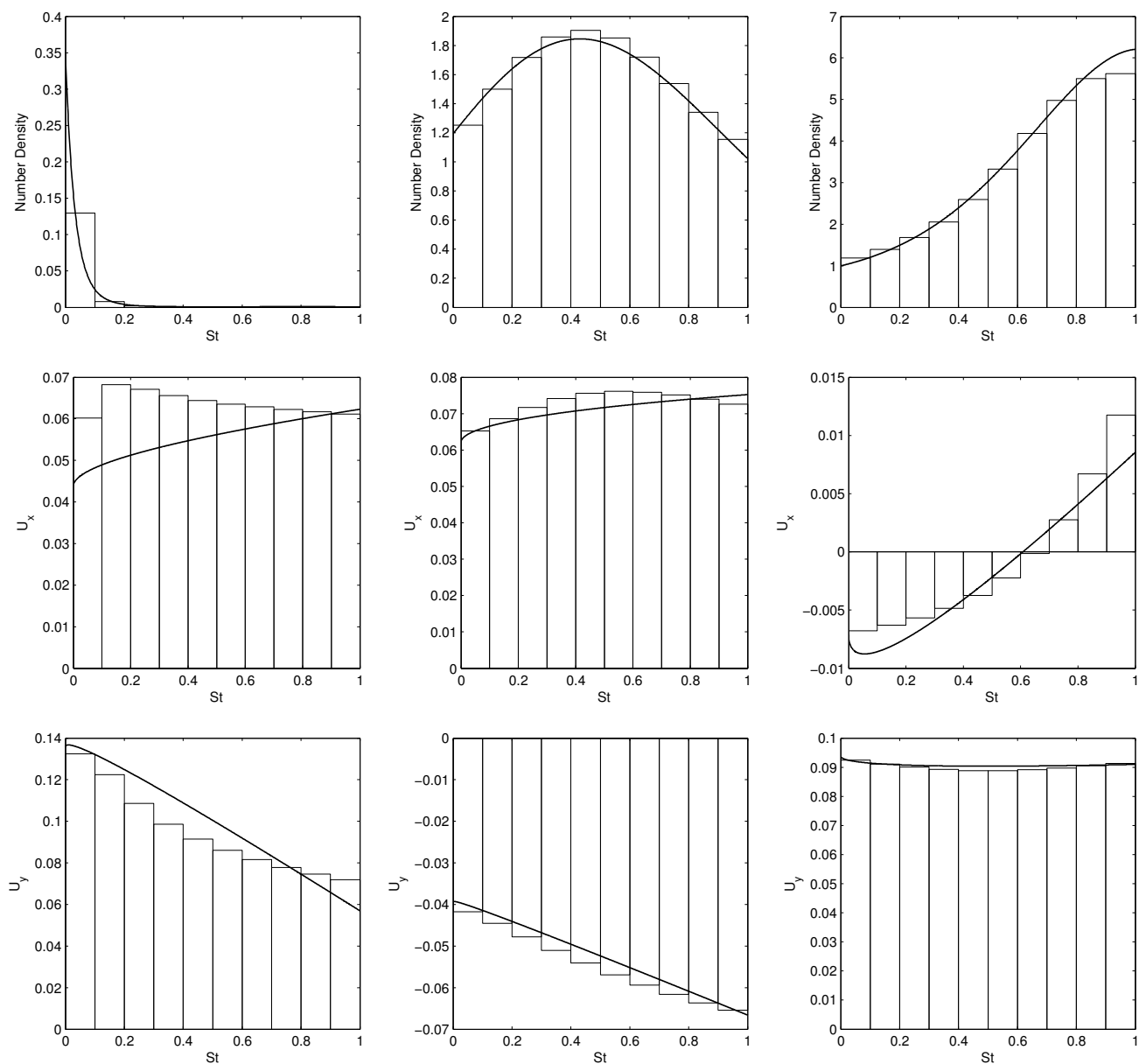

Figure 4. Number density functions (up), velocity in $x$ direction (middle) and in $y$ direction (down) at specific positions in the domain: Multifluid 10 sections (histograms) and CSVM (full line) inside a vortex (left), in a medium number density zone (center), and in a high density zone (right). 


\section{Conclusions and perspectives}

In this work, the CSVM method has been evaluated on a Frozen Homogeneous Isotropic Turbulence. Results demonstrate the ability of this approach to achieve the same accuracy of a Multifluid method, using only 8 moments comparing to the 30 moments of the Multifluid. All essential features of the flow, such as the segregation or the spatial mean size variance are well captured, which is not possible with the EMSM which does not take into account the size-conditioned dynamics.

Now, to reach the implementation of such methods in industrial codes, some issues need to be handled. First a second order scheme is needed, and the scheme proposed in [21] will be tested. Second, the method has to be adapted to turbulent flows, for instance by coupling with the MEF of Février et al. [2]. Third, the computational cost of such method needs to be optimized, by using a more efficient way for evaluating integrals.

Then, the CSVM will be implemented in parallel solvers like MUSES3D to evaluate the method on 3D reactive configurations to assess its efficiency on flame dynamics and auto-ignition. Finally, it will be implemented in industrial codes such as AVBP or IFP-C3D, and will be applied on real complex configurations, like diesel injection or aeronautical burners.

\section{Acknowledgments}

We would like to thanks Damien Kah for several helpful discussions and for providing numerous inputs and code sources from his Ph.D. Thesis where the EMSM was introduced. The motivation for the present study also stems from an original study of the crossflow configuration conducted in the Ph.D. Thesis of A. Vié as well as from discussions with his advisers S. Jay (IFPEN) and B. Cuenot (CERFACS), whom we take the occasion to thank here. The support from the RTRA DIGITEO as well as from Ecole Centrale Paris through the "Direction de la Recherche" for the Post-doctoral fellowship for A. Vié is gratefully acknowledged. In fact the present study received a funding from this RTRA through the MUSE project (PI M. Massot - "MUltiscale Spray combustion fully Eulerian solver in 3D : a new generation of numerical methods and algorithms, high performance simulations, validation and visualization").

\section{References}

[1] D. Kah. Taking into account polydispersity in the framework of a coupled Euler-Lagrange approach for the modeling of liquid fuel injection in internal combustion engines. $\mathrm{PhD}$ thesis, Ecole Centrale de Paris, 2010. Available online at http://tel.archives-ouvertes.fr/tel-00618786/en/.

[2] P. Février, O. Simonin, and K. D. Squires. Partitioning of particle velocities in gas-solid turbulent flow into a continuous field and a spatially uncorrelated random distribution: theoretical formalism and numerical study. Journal of Fluid Mechanics, 533:1-46, 2005.

[3] M. Boileau, C. Chalons, F. Laurent, S. de Chaisemartin, and M. Massot. Robust numerical schemes for eulerian spray DNS and LES in two-phase turbulent flows. Proceedings of the Summer Program 2010. Center for Turbulence Research, Stanford, pages 359-370, 2011.

[4] E. Masi, O. Simonin, and B. Bédat. The mesoscopic eulerian approach for evaporating droplets interacting with turbulent flows. Flow, Turbulence and Combustion, 86:563-583, 2011.

[5] J. Dombard. Direct Numerical Simulation of non-isothermal dilute sprays using the Mesoscopic Eulerian Formalism. PhD thesis, Institut National Polytechnique de Toulouse, 2011.

[6] O. Desjardin, R.O. Fox, and P. Villedieu. A quadrature-based moment method for dilute fluid-particle flows. J. Comput. Phys., 227:2514-2539, 2008. 
[7] C. Yuan and R.O. Fox. Conditional quadrature method of moments for kinetic equations. Journal of Computational Physics, 230(22):8216-8246, 2011.

[8] C. Chalons, D. Kah, and M. Massot. Beyond pressureless gas dynamics: quadrature-based velocity moment models. Communication in Mathematical Sciences, 10(4):1241-1272, 2012. Available online at http://hal.archives-ouvertes.fr/hal-00535782/en.

[9] C. Chalons, R. O. Fox, and M. Massot. A multi-Gaussian quadrature method of moments for gas-particle flows in a LES framework. In Proceedings of the Summer Program 2010, Center for Turbulence Research, Stanford University, pages 347-358, Stanford, 2010.

[10] A. Vié, C. Chalons, R. O. Fox, F. Laurent, and M. Massot. A multi-Gaussian quadrature method of moments for simulating high-stokes-number turbulent two-phase flows. Annual Research Briefs, Center for Turbulence Research, pages 309-320, 2011.

[11] F. Laurent and M. Massot. Multi-fluid modeling of laminar poly-dispersed spray flames: origin, assumptions and comparison of the sectional and sampling methods. Combust. Theory and Modelling, 5:537-572, 2001.

[12] S. de Chaisemartin. Eulerian models and numerical simulation of turbulent dispersion for polydisperse evaporation sprays. PhD thesis, Ecole Centrale Paris, France, 2009. Available on TEL : http://tel.archives-ouvertes.fr/tel-00443982/en/.

[13] L. Fréret, O. Thomine, J. Reveillon, S. de Chaisemartin, F. Laurent, and M. Massot. On the role of preferential segregation in flame dynamics in polydisperse evaporating sprays. In Proceedings of the Summer Program 2010, Center for Turbulence Research, Stanford University, pages 383-392, 2011.

[14] D.L. Marchisio, J.T. Pikturna, R.O. Fox, and R.D. Vigil. Quadrature method of moments for population-balance equations. AIChE Journal, 49(5):1266-1276, 2003.

[15] R.O. Fox, F. Laurent, and M. Massot. Numerical simulation of spray coalescence in an eulerian framework: Direct quadrature method of moments and multi-fluid method. Journal of Computational Physics, 227:3058-3088, 2008.

[16] D. Kah, F. Laurent, M. Massot, and S. Jay. A high order moment method simulating evaporation and advection of a polydisperse spray. J. Comput. Phys., 231(2):394-422, 2011.

[17] M. Massot, F. Laurent, D. Kah, and S. de Chaisemartin. A robust moment method for evaluation of the disappearance rate of evaporating sprays. SIAM J. Appl. Math., 70:3203-3234, 2010.

[18] J.B. Mossa. Extension polydisperse pour la description Euler-Euler des écoulements diphasiques réactifs. PhD thesis, Institut National Polytechnique de Toulouse, 2005.

[19] D. Kah, F. Laurent, L. Fréret, S. de Chaisemartin, R.O. Fox, J. Reveillon, and M. Massot. Eulerian quadrature-based moment models for dilute polydisperse evaporating sprays. Flow, Turbulence and Combustion, 85(3-4):649-676, 2010.

[20] A. Vié, S. Jay, B. Cuenot, and M. Massot. Accounting for polydispersion in the eulerian large eddy simulation of an aeronautical-type configuration. Submitted to Flow Turbulence and Combustion, 2012. Available on HAL at http://hal.archives-ouvertes.fr/hal-00650605/.

[21] A. Vié, F. Laurent, and M. Massot. Size-velocity correlations in high order moment methods: modelling and numerical issues. Submitted to J. Comput. Phys., 2012. Available online at http://hal.archives-ouvertes.fr/hal-00626869/en/.

[22] L. R. Mead and N. Papanicolaou. Maximum entropy in the problem of moments. J. Math. Phys., $25(8): 2404-2417,1984$.

[23] D.L. Wright. Numerical advection of moments of the particule size distribution in eulerian models. Journal of Aerosol Science, 38(3):352-369, 2007.

[24] S. B. Pope. Turbulent Flows. Cambridge University Press, Cambridge, 2000. 